\title{
Dynamical phase transitions in long-range Hamiltonian systems and Tsallis distributions with a time-dependent index
}

\author{
Alessandro Campa, ${ }^{1}$ Pierre-Henri Chavanis, ${ }^{2}$ Andrea Giansanti, ${ }^{3}$ and Gianluca Morelli ${ }^{4}$ \\ ${ }^{1}$ Theoretical Physics Unit, Health and Technology Department, Istituto Superiore di Sanità, \\ and INFN Roma1, Gruppo Collegato Sanità, Viale Regina Elena 299, 00161 Roma, Italy \\ ${ }^{2}$ Laboratoire de Physique Théorique - IRSAMC, CNRS Université Paul Sabatier, 31062 Toulouse, France \\ ${ }^{3}$ Physics Department, Università di Roma "La Sapienza", Piazzale Aldo Moro 2, 00185 Roma, Italy \\ ${ }^{4}$ Physics Department, Università di Roma "La Sapienza" Piazzale Aldo Moro 2, 00185 Roma, Italy
}

(Dated: November 1, 2018)

\begin{abstract}
We study dynamical phase transitions in systems with long-range interactions, using the Hamiltonian Mean Field (HMF) model as a simple example. These systems generically undergo a violent relaxation to a quasi-stationary state (QSS) before relaxing towards Boltzmann equilibrium. In the collisional regime, the out-of-equilibrium one-particle distribution function (DF) is a quasistationary solution of the Vlasov equation, slowly evolving in time due to finite $N$ effects. For subcritical energy densities, we exhibit cases where the DF is well-fitted by a Tsallis $q$-distribution with an index $q(t)$ slowly decreasing in time from $q \simeq 3$ (semi-ellipse) to $q=1$ (Boltzmann). When the index $q(t)$ reaches an energy dependent critical value $q_{\text {crit }}$, the non-magnetized (homogeneous) phase becomes Vlasov unstable and a dynamical phase transition is triggered, leading to a magnetized (inhomogeneous) state. While Tsallis distributions play an important role in our study, we explain this dynamical phase transition by using only conventional statistical mechanics. For supercritical energy densities, we report for the first time the existence of a magnetized QSS with a very long lifetime.
\end{abstract}

PACS numbers: 05.20.-y, 05.10.-a, 05.70.Ln

Keywords: Long-range interactions; Quasi Stationary States; Lynden-Bell theory; Tsallis distributions

Systems with long-range interactions have a peculiar dynamics and thermodynamics [1]. In these systems, the potential of interaction decreases at large distances like $r^{-\alpha}$, with an exponent $\alpha$ smaller than the dimension of space. As a result, the energy is non-additive and this can lead to negative specific heats and inequivalence of statistical ensembles. The equilibrium properties of these systems are now well-understood and the main challenge concerns the description of their out-of-equilibrium properties. The general methodology to study these systems can have impact in different domains of physics (see various contributions in 1]).

Long-range systems can spontaneously develop outof-equilibrium coherent structures, called quasi stationary states (QSS). The QSS are generically characterized by non-Boltzmannian distributions and their lifetime diverges with the size $N$ of the system. Galaxies in the universe and $2 \mathrm{D}$ vortices in geophysical and astrophysical flows are examples of such QSS [2]. They are also observed in non-neutral plasmas under a strong magnetic field [3], in the free electron laser [4] and in simple models like the Hamiltonian mean-field (HMF) model [5, 6, 7, 8, 9, 10]. They are therefore general features of systems with long-range interactions. The interesting issues about the nature of these QSSs concern: i) their rapid formation starting from a generic non equilibrium initial condition; ii) their structure and stability; iii) their slow evolution due to finite $N$ effects; iv) their final transition to the Boltzmann equilibrium distribution. In particular, point iv) requires to develop a theory of dynamical phase transition adapted to these systems.

In this perspective, the HMF model [1] became a paradigmatic laboratory. It represents a system of $N$ globally coupled rotators of unit mass described by the Hamiltonian:

$$
H=\frac{1}{2} \sum_{i=1}^{N} p_{i}^{2}+\frac{1}{2 N} \sum_{i, j=1}^{N}\left[1-\cos \left(\theta_{i}-\theta_{j}\right)\right],
$$

where $\theta_{i}$ represents the orientation of the $i$-th rotator and $p_{i}$ is the conjugated momentum. The evolution of the system may be followed through the magnetization, an order parameter defined as $M=|\mathbf{M}|=\left|\sum \mathbf{m}_{i}\right| / N$, where $\mathbf{m}_{i}=\left(\cos \theta_{i}, \sin \theta_{i}\right)$. The HMF model has been extensively studied as a representative of a broad class of systems with long-range interactions, like gravitational systems (see references in [7]). The equilibrium solution [11] reveals the existence of a second-order phase transition at the critical energy density $U_{c}=3 / 4$ : the Boltzmann equilibrium state is homogeneous (non-magnetized) for $U>U_{c}$ and inhomogeneous (magnetized) for $U<U_{c}$.

On the other hand, the interpretation of QSS is an issue of tense debate that has recently polarized researchers in two groups. The first group [5] interprets the QSS as non-Boltzmannian equilibrium states explainable within Tsallis generalized thermodynamics [12] when the thermodynamic limit $N \rightarrow+\infty$ is taken before the infinite time limit $t \rightarrow+\infty$. The justification advocated is that the evolution of the system is generally non-ergodic so that non-standard distributions can emerge. The other group [6, 7, 8, 9] interprets the QSS in terms of the 
Lynden-Bell [13] theory of violent relaxation that was initially developed for collisionless stellar systems and 2D vortices [2]. For sufficiently short times, or for $N \rightarrow+\infty$, the evolution of the one-particle distribution function (DF) is governed by the Vlasov equation, a mean field equation that ignores correlations between particles [14]. In this context, the QSS result from a process of phase mixing and violent relaxation on a coarse-grained scale. The statistical theory of Lynden-Bell [13] predicts the most mixed state consistent with all the constraints of the collisionless (Vlasov) dynamics.

Numerical simulations have given contrasting results. For a water-bag initial condition (i.e., uniform distribution of velocities and angles in an interval symmetric around 0 ) with energy density $U=0.69$, it has been found that the Lynden-Bell theory predictions are correct [9] if the initial value of the magnetization $M_{0} \equiv M(t=$ $0)$ is below a critical value $\left(M_{0}\right)_{\text {crit }}=0.897$ appearing in the theory [8, [9], while this is no more true if $M_{0}$ is close to 1 [5, 10]. In particular, for $M_{0}=1$, Campa et al. [10] find that the QSS is well-fitted by a homogeneous $\left(M_{Q S S}=0\right)$ semi-elliptical distribution, which is a particular Tsallis DF with $q=3$ (as noted in 14]), while the Lynden-Bell prediction is an inhomogeneous $\left(M_{Q S S} \neq 0\right)$ Boltzmann distribution corresponding to the non degenerate limit of the theory [14, 15]. Stimulated by these results, one of us [8] has proposed an approach in which the two points of view can be reconciled in the following manner: (i) if the system mixes well (ergodicity), the QSS is (close to) the "most mixed state" predicted by Lynden-Bell's theory; (ii) if the system does not mix well (non ergodicity), relaxation is incomplete and nonstandard DFs (including the important class of Tsallis distributions) can occur.

The purpose of this Rapid Communication is to give numerical evidence that Tsallis distributions can also characterize the slow collisional evolution of the QSS (due to finite $N$ effects) up to the relaxation to Boltzmann equilibrium. Our results support the proposal made in Ref. 14], according to which the out-of-equilibrium DF, for a large class of initial conditions, could be fitted by a Tsallis $q$-distribution (see below) with an index $q(t)$ depending on time. We will show that the dynamical phase transition from the non-magnetized QSS to the magnetized Boltzmann distribution is triggered when the value $q(t)$ is such that the DF becomes Vlasov unstable: therefore, the final transition to the Boltzmann distribution is driven by a dynamical instability with respect to the Vlasov equation.

In a non-magnetized QSS, the DF depends only on velocity and slowly evolves in time due to finite $N$ effects. It turns out that the time-dependent DF is very well fitted by Tsallis distributions (polytropes):

$$
f(v, t)=C(t)\left[1-\left(\frac{v}{v_{\max }(t)}\right)^{2}\right]^{1 /(q(t)-1)}
$$

with a time dependent index $q(t) \geq 1$. The support of the function is the interval $\left[-v_{\max }(t), v_{\max }(t)\right]$; the normalization factor $C(t)$ and $v_{\max }(t)$ depend on time through $q(t)$. The normalization condition $\int_{-\infty}^{+\infty} f d v=1 /(2 \pi)$ and the conservation of energy $U-1 / 2=\pi \int_{-\infty}^{+\infty} f v^{2} d v$ lead to the relations $1 /(2 \pi)=C v_{\max } \sqrt{\pi} \Gamma(1+1 /(q-$ 1)) $/ \Gamma(3 / 2+1 /(q-1))$ and $U-1 / 2=\pi C v_{\max }^{3}(\sqrt{\pi} / 2) \Gamma(1+$ $1 /(q-1)) /\{(\Gamma(3 / 2+1 /(q-1))(3 / 2+1 /(q-1))\}$ from which we obtain the identity $v_{\max }^{2}(t)=4(U-1 / 2)(3 / 2+$ $1 /(q(t)-1))$. On the other hand, using the stability criterion $1+\pi \int_{-\infty}^{+\infty}\left(f^{\prime}(v) / v\right) d v \geq 0$ of [6, 16] (see also [7]) we find that a spatially homogeneous $q$-distribution is Vlasov stable (dynamical stability) iff $U \geq U_{\text {crit }}(q) \equiv$ $1 / 2+(1 / 4)(q+1) /(3 q-1)$ [7, 14, 17]. For $q=1$ (Boltzmann) we recover $U_{c r i t}(1)=U_{c}=3 / 4$, for $q \rightarrow+\infty$ (water-bag) we recover $U_{*} \equiv U_{\text {crit }}(\infty)=7 / 12$ and for $q=3$ (semi-elliptical), we recover $U_{\text {crit }}(3)=5 / 8$ 10]. From this criterion, DFs of the form (2) are always stable for $U>U_{c}=3 / 4$ and always unstable for $U<U_{*}=7 / 12$. For $U_{*}<U<U_{c}$, they are stable iff $q>q_{\text {crit }}(U)$ with [18]

$$
q_{\text {crit }}(U)=\frac{4 U-1}{12 U-7}
$$

Campa et al. [10] considered a water-bag initial condition with $M_{0}=0$ at energy density $U=0.69$. This initial $\mathrm{DF}$ is already a maximum Lynden-Bell entropy state so it does not experience phase mixing and violent relaxation 14]. However, it slowly evolves due to finite $N$ effects. In Ref. 10 it was found that the system rapidly forms a velocity DF of semi-elliptical shape. As we have already indicated, this is a Tsallis DF of the form (2) with $q=3$. However, the slow evolution of the DF on longer times was not quantitatively addressed. Here, we have performed simulations at several energies, all of them starting from an unmagnetized state (uniform angle distribution). For the subcritical energy densities $U<U_{c}$, the initial velocity distribution was a semi-elliptical, $q=3$, function. The energy densities were all above the critical value $5 / 8$ corresponding to $q=3$, and therefore the initial steady states were Vlasov stable. In these cases we found that during the QSS, while the magnetization remains zero (or, more precisely, oscillates about a value scaling to zero as $1 / \sqrt{N}$ because of finite size effects), the velocity $\mathrm{DF}$ has a slow evolution. At a certain point the distribution is no more Vlasov stable; then the magnetization begins to increase relatively fast, and the systems heads towards Boltzmann equilibrium. During the slow process characterizing the QSS, we found that the velocity DF remains always close to one of the $q$ functions (2), so that it is possible to define a function of time $q(t)$. In this way, the evolution of the velocity DF is described by the slow decay of a single quantity, the index $q(t)$ of the polytrope, from the value $q(0)=3$ to the energy dependent critical value $q_{\text {crit }}(U)$. 
In Fig. 1, we show results corresponding to $U=0.69$ (similar results, not shown, were obtained for $U=0.65$, $U=0.67$, and $U=0.71$ ). The left panel shows the evolution of the magnetization $M(t)$, while the right panel shows the evolution of the function $q(t)$. The runs correspond to $N=2^{14}$. The critical value of $q$ corresponding to $U=0.69$ is $q_{\text {crit }}(0.69)=1.375$. This value is indicated, for reference, as a dashed line in the graph of $q(t)$. At each time, the best value of $q$ is determined by a least square procedure, minimizing with respect to $q$ the sum of the squares of the differences between the numerical distribution and the $q$-distribution for the energy density $U=0.69$. In this procedure, both the numerical and the theoretical distributions are given as histograms with a suitably chosen bin width; $q$ is varied in steps of 0.001 . We see that the destabilization of the DF, and the associated process of magnetization of the system, is related to the approach of $q(t)$ towards the critical value $q_{\text {crit }}(U)$. We found that this behavior is present for all $N$ values that we have studied, although noise obviously increases if $N$ is decreased.
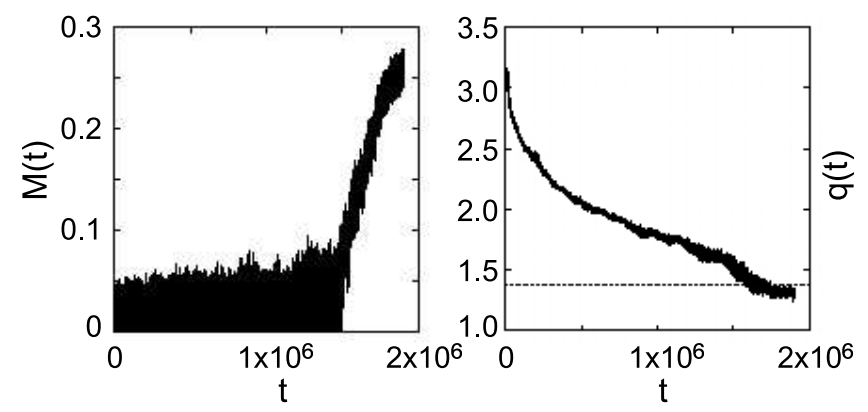

FIG. 1: Time evolution of the magnetization $M(t)$ (left panel) and of the index $q(t)$ (right panel), for $U=0.69$ and $N=2^{14}$. The dotted line in the right panel indicates the critical value $q_{\text {crit }}(0.69)=1.375$. During the collisional process, the DF is a quasi-stationary solution of the Vlasov equation of the form (2) slowly evolving in time due to finite $N$ effects (see Figure 2 ). When $q(t)$ reaches $q_{\text {crit }}$, it becomes dynamically (Vlasov) unstable and the phase transition is triggered.

In Fig. 2 we show snapshots of the DF during the "collisional" relaxation for the same run as that shown in the previous figure. The full lines give the numerical distributions at a given time, and the dotted lines are the $q$-functions for the best fitted value of $q(t)$. The fit by a Tsallis DF with a time dependent index is very good during all the out-of-equilibrium evolution.

Finally, we consider the case of a supercritical energy density $U>U_{c}=3 / 4$. For $U=0.8$, Campa et al. [10] started from a non-magnetized state with a semielliptical distribution $(q=3)$, which is Vlasov stable, and found that the system relaxes to the Boltzmann equilibrium distribution on a very slow timescale scaling like $t_{\text {coll }}(N) \sim e^{N}$. Since $q_{\text {crit }}(U)<1$ for $U>U_{c}$, while Tsallis distributions with a compact support have $q(t)>1$,
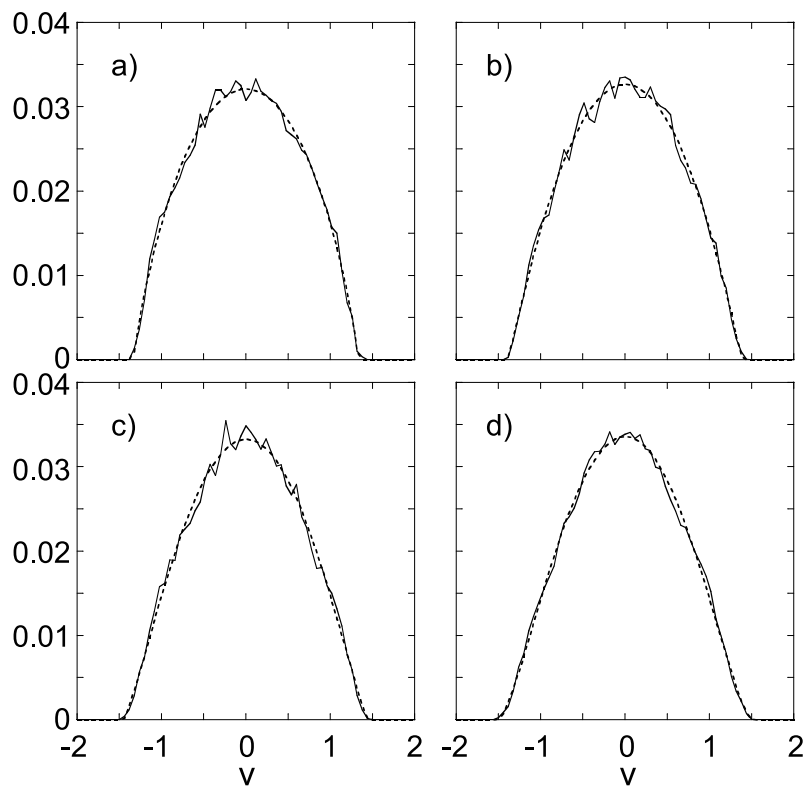

FIG. 2: Velocity DF at four different times during the QSS of the run shown in Figure 1. The full lines are the numerical distributions, while the dotted lines are the $q$-functions for the best fitted value of $q(t)$. a) $t=3 \times 10^{5}$ and $q(t)=2.224$; b) $t=6 \times 10^{5}$ and $q(t)=1.987$; c) $t=9 \times 10^{5}$ and $q(t)=1.789$; d) $t=1.2 \times 10^{6}$ and $q(t)=1.697$. The absolute value of the DF depends on the chosen bin width.

the spatially homogeneous phase remains always Vlasov stable during the collisional evolution and no dynamical phase transition occurs. This explains [14] the long observed relaxation time for $U>U_{c}$ as compared to the case $U<U_{c}$. In this case, the relaxation is due to a slow collisional relaxation, not to a dynamical (Vlasov) instability. Considering a slightly different supercritical energy density, $U=0.85$, for which $q_{\text {crit }}=0.75$, the left panel of Fig. 3 shows the function $q(t)$ that, starting from $q(0)=3$, slowly approaches 1 . During this relaxation the DF is well approximated, as in the subcritical case shown in Fig. 2, by the $q$-function with $q=q(t)$. We have also studied another type of initial condition, at the supercritical energy density $U=0.85$, starting from a non-magnetized state with a Tsallis velocity DF corresponding to $q=0.5$, which is below the critical value 0.75. This initial DF is Vlasov unstable, linear instability followed by phase mixing and violent relaxation quickly drive the system towards a magnetized out-ofequilibrium QSS. Although the system should eventually relax to a non-magnetized Boltzmann distribution, the right panel of Fig. 3, corresponding to a run with $N=2^{11}$, shows that the duration of this magnetized QSS is extraordinary long (probably exponential in $N$, as estimated by runs at different $N$ values). For comparison, we note that for $N=2^{11}$ the QSS at $U=0.69$ lasts for a time of about $5 \times 10^{4}[10]$. 
In conclusion, we have shown that Tsallis $q$ distributions (polytropes) can be useful to describe certain features of the complex HMF dynamics. First, they can provide good fits of the QSS in certain cases of incomplete relaxation [10, 14]. However, this is not general 14] and other DF that are stable stationary solutions of the Vlasov equation can be observed as well (the system can also have a non-stationary oscillatory behaviour [19]). More interestingly, like in the gravitational simulations of Taruya and Sakagami 20], we have found that the out-of-equilibrium evolution of the DF in the collisional regime can be fitted by Tsallis distributions with a time dependent index $q(t)$. This may be a general feature of the dynamics of long-range systems. However, while Taruya and Sakagami 20] interpret the dynamical phase transition as a generalized thermodynamical instability (in Tsallis sense), we interpret it as a dynamical instability with respect to the Vlasov equation [14, 21].
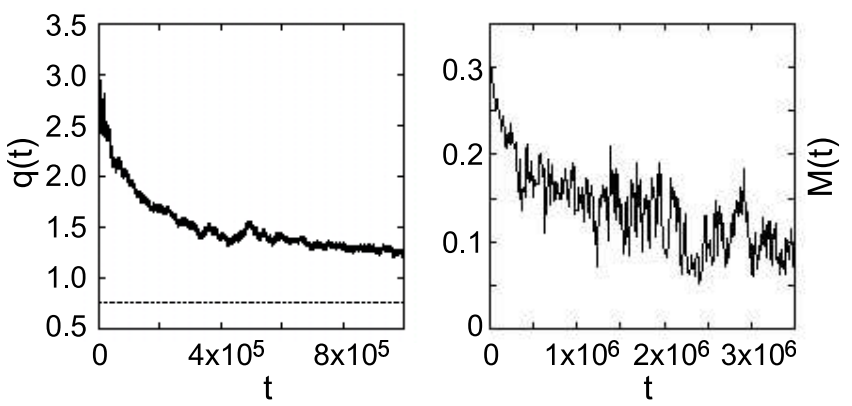

FIG. 3: Time evolution of the index $q(t)$ (left panel) and of the magnetization $M(t)$ (right panel), for $U=0.85$ and $N=2^{11}$. The left panel refers to a run with $q(0)=3$, in which the system remains always demagnetized $\left(q(t)>q_{\text {crit }}=0.75\right)$ and slowly approaches Boltzmann equilibrium $(q=1)$; the right panel refers to a run with $q(0)=0.5<q_{\text {crit }}=0.75$, in which the QSS is magnetized and lasts for a very long time.

The evolution of the system from the QSS to the Boltzmann distribution, due to the development of correlations ("collisions") between particles, could be understood by developing a kinetic theory of the Hamiltonian $N$-body problem. As a matter of fact, starting from the Liouville equation and using an expansion of the solutions of the BBGKY-like hierarchy in powers of $1 / N$ in a proper thermodynamic limit $N \rightarrow+\infty$, a general kinetic equation can be obtained at the order $O(1 / N)$ [14]; the BBGKY hierarchy is closed, neglecting three body and higher correlations of order $1 / N^{2}$ or smaller. However, for one dimensional systems like the HMF model, this kinetic equation does not tend to the Boltzmann distribution (for spatially homogeneous systems the collision term, which reduces to the Landau or Lenard-Balescu operator [14, 22], cancels out identically in $1 D$ ). This implies that higher order correlations are needed to understand the convergence of the system to the Boltzmann distribution. Therefore, we can only conclude that the colli- sional relaxation time $t_{\text {coll }}(N)$ is larger than $N t_{D}$ (where $t_{D}$ is a typical dynamical time of the system), but its precise scaling with $N$ cannot be deduced from the present kinetic theory. Different scalings have been found numerically depending on the initial condition: $N t_{D}$ [5], $N^{1.7} t_{D}$ [6, 10] and $e^{N} t_{D}$ [10]. Due to the considerable timescale separation between the dynamical time (of order $t_{D}$ ) and the collision time (larger than $N t_{D}$ ), it can be argued that, during the slow "collisional" relaxation, the time dependent DF remains close to a steady solution of the Vlasov equation that slowly evolves under the effect of collisions (finite $N$ effects). In this Rapid Communication we have shown that, at least for a class of initial conditions, these slowly evolving QSS are wellfitted by Tsallis $q(t)$-functions. However, as discussed in Sec. 5 of 21] this is not necessarily the mark of a "generalized thermodynamics" [12]. Indeed, if using only classical methods of kinetic theory (consistent with the Boltzmann entropy), we were able to write down the kinetic equation describing the collisional relaxation of the spatially homogeneous HMF model (the analogue of the orbit-averaged-Fokker-Planck equation in astrophysics), the DF (2) could form an approximate time dependent solution of this classical kinetic equation (without relation to generalized thermodynamics). Unfortunately, for the HMF model, this kinetic equation is not known.

[1] Dynamics and thermodynamics of systems with long range interactions, edited by $\mathrm{T}$. Dauxois et al., Lecture Notes in Physics 602 (Springer, 2002); Dynamics and thermodynamics of systems with long range interactions: Theory and experiments, edited by A. Campa et al., AIP Conf. Proc. 970 (AIP, 2008).

[2] P.H. Chavanis et al., Astrophys. J. 471, 385 (1996).

[3] X. P. Huang and C. F. Driscoll, Phys. Rev. Lett. 72, 2187 (1994).

[4] J. Barré et al., Phys. Rev. E 69, 045501 (2004).

[5] V. Latora et al., Phys. Rev. E 64, 056134 (2001).

[6] Y. Yamaguchi et al., Physica A 337, 36 (2004).

[7] P.H. Chavanis et al., Eur. Phys. J. B 46, 61 (2005).

[8] P.H. Chavanis, Eur. Phys. J. B 53, 487 (2006).

[9] A. Antoniazzi et al., Phys. Rev. E 75, 011112 (2007).

[10] A. Campa et al., Phys. Rev. E 76, 041117 (2007).

[11] M. Antoni and S. Ruffo, Phys. Rev. E 52, 2361 (1995).

[12] C. Tsallis, J. Stat. Phys. 52, 479 (1988).

[13] D. Lynden-Bell, Mon. Not. R. Astron. Soc. 136, 101 (1967).

[14] P.H. Chavanis, Physica A 387, 787 (2008).

[15] As pointed out in [8, 14], the failure of the Lynden-Bell prediction for $M_{0}=1$ may be due to the proximity of this value to the critical magnetization $\left(M_{0}\right)_{\text {crit }}=0.897$, very close to 1 for the energy density $U=0.69$.

[16] S. Inagaki and T. Konishi, Publ. Astron. Soc. Jpn. 45, 733 (1993).

[17] C. Anteneodo and R. Vallejos, Physica A 344, 383 (2004).

[18] Below this critical value, the DF (2) ceases to be a max- 
imum of the Casimir functional $S_{q}=-1 /(q-1) \int\left(f^{q}-\right.$ $f) \mathrm{d} \theta \mathrm{d} v$ (at fixed mass and energy) and it becomes a saddle point [6, 7].

[19] H. Morita and K. Kaneko, Phys. Rev. Lett. 96, 050602 (2006).
[20] A. Taruya and M. Sakagami, Phys. Rev. Lett. 90, 181101 (2003).

[21] P.H. Chavanis and C. Sire, Physica A 356, 419 (2005).

[22] F. Bouchet, T. Dauxois, Phys. Rev. E 72, 045103 (2005). 